(2) OPEN ACCESS

\title{
Microvascular abnormalities and long-term efficacy after stereotactic radiotherapy under continued intravitreal anti-VEGF treatment for neovascular AMD
}

\author{
Katja Hatz 이 , 1,2 Frank Zimmermann, ${ }^{3}$ Emmanouil Lazaridis, ${ }^{4}$ Dimitrios Kardamakis, ${ }^{5}$ \\ Magdalena Guichard, ${ }^{1}$ Cengiz Türksever, ${ }_{1}^{1}$ Christian Pruente, ${ }^{6,7}$ \\ Ursula Margarethe Schmidt-Erfurth (10, ${ }^{8}$ Bianca S Gerendas (i) ${ }^{8}$
}

\begin{abstract}
- Additional material is published online only. To view please visit the journal online (http://dx.doi.org/10.1136/ bjophthalmol-2020-317563).

${ }^{1}$ Vista Klinik, Binningen, Switzerland

'University of Basel Faculty of Medicine, Basel, BS, Switzerland ${ }^{3}$ Department of Radiation Oncology, University Hospital Basel, Basel, BS, Switzerland ${ }^{4}$ EyeRAD Swiss Medical Center, Binningen, Switzerland ${ }^{5}$ Department of Radiation Oncology, University of Patras Department of Medicine, Patras, Achaea, Greece

${ }^{6}$ Department of Ophthalmology, University of Basel, Basel, BS, Switzerland

${ }^{7}$ Institute of Molecular and Clinical Ophthalmology, Basel, Switzerland

${ }^{8}$ Department of Ophthalmology and Optometry, Medical University of Vienna, Wien, Austria
\end{abstract}

Correspondence to Dr Katja Hatz, Vista Klinik, Binningen, Binningen, Switzerland;

katja.hatz@unibas.ch

Received 28 July 2020 Revised 23 October 2020 Accepted 30 October 2020

\begin{abstract}
Background For treatment of neovascular age-related macular degeneration (nAMD), multiple intravitreal injections of drugs targeting vascular endothelial growth factors (VEGF) result in a high burden for patients and healthcare systems. Low-energy stereotactic radiotherapy (SRT) might reduce the anti-VEGF need. This study evaluated the long-term efficacy and safety of adjunct SRT to anti-VEGF injections in a treat-and-extend regimen in $\mathrm{nAMD}$.
\end{abstract}

Methods 50 consecutive patients were followed 3 years after single-session SRT, a safety analysis including standardised study imaging, and a reading centre based image analysis was performed after 2 years.

Results After increase from baseline $(4.24 \pm 0.66$ weeks) to 12 months $(7.52 \pm 3.05$ weeks, $p<0.001)$, mean recurrence-free anti-VEGF treatment interval remained stable at $24(7.40 \pm 3.17, p=0.746)$ and 36 months $(6.89 \pm 3.00, p=0.175)$. Mean visual acuity change was $-5.8 \pm 15.9$ and $-11.0 \pm 20.1$ letters at 24 and 36 months, respectively. $36 \%$ of eyes showed microvascular abnormalities (MVAs) on colour fundus photography and/or fluoresceine angiography most frequently located in parafoveal inferior and nasal regions.

Conclusion In real life, low-energy SRT was associated with a reduced anti-VEGF injection frequency through year 3. However, due to an observed visual acuity reduction and remarkable number of MVAs, a close follow-up of these patients is recommended. The reallife use, optimal treatment schedule and dose should be rediscussed critically.

\section{INTRODUCTION}

Age-related macular degeneration (AMD) is the leading cause of severe visual impairment in individuals over 50 years in developed countries. ${ }^{1}$ Intravitreal injections of antivascular growth factor (anti-VEGF) substances have become the gold standard in treatment of neovascular AMD (nAMD). ${ }^{2}$ Despite an improved prognosis, ${ }^{2}$ in the real world, the visual acuity outcomes are often disappointing due to insufficient frequency of follow-up/treatments. ${ }^{3}$ Continuous injecting schemes like fixed ${ }^{4}$ or the treat-and-extend regimen (TER) ${ }^{56}$ provide better visual acuity results compared with the widely used pro re nata (PRN) regimen. However, beside the high burden for the patients, the costs are enormous. ${ }^{7-9}$ Therefore, different adjunct treatments aiming to reduce anti-VEGF treatment frequency have been considered, such as verteporfin photodynamic therapy ${ }^{10} 11$ or radiation therapy. ${ }^{12-17}$

Generally, there are two types of radiotherapy being reported in nAMD: the external and the internal type. In the external type, called teletherapy, an individually conformed radiation beam of an external source is projected into the target tissue. $^{18} 19$ Stereotactic radiotherapy (SRT) is a special type of teletherapy using multiple narrow beams of radiation (kilovoltage or megavoltage) being used to target small, well-defined areas with high precision under perfect positioning of the patient and using navigational systems as frames or imaging, such as the IRay System (Carl Zeiss Meditec AG Germany). ${ }^{15-17}$ In the internal radiotherapy type, called epimacular brachytherapy (EMBT), a radiation source is directly placed next to the target site surgically. ${ }^{12-14}$ In a few studies, EMBT combined with anti-VEGF injections led to a reduction of anti-VEGF retreatments, ${ }^{12-14}$ while another study failed to show superiority of EMBT regarding fewer injections. ${ }^{20}$ In contrast, SRT as a special type of external radiotherapy significantly reduced the number of anti-VEGF injections compared with SRT-sham treatment in the INTREPID (IRay Plus Anti-VEGF Treatment For Patients With Wet AMD) trial. ${ }^{16}$ During a short-term follow-up, it seems that the combination of the more strict anti-VEGF TER and SRT could prevent the vision loss as shown in INTREPID while significantly lengthening the antiVEGF treatment interval. ${ }^{17}$ However, it remains unclear if this effect persists over a long term.

In the reported trials, the rate and extension of side effects was different, probably due to the different approaches reflecting radiotherapy side effects. ${ }^{18}$ Radiation retinopathy (RR) is the most dreaded long-term complication of radiotherapy to the eye. It has extensively been studied in patients with ocular tumours ${ }^{21}$ but was also shown in nAMD radiation eyes; for both approaches, EMBT and SRT, the rate of patients with microvascular abnormalities (MVAs) as signs of RR were low until end of year 2. ${ }^{13} 141620$

However, evaluation of MVAs due to radiotherapy for AMD followed different methods within these trials. Mostly the fields evaluated by fluoresceine angiography and/or colour fundus 
photography were not described in detail, and the authors exclusively reported central results regarding MVAs. ${ }^{1622} 23$ Due to the moment of setup of these trials, time-domain optical coherence tomography (OCT) was used instead of spectral-domain (SD) OCT. Grading was performed by reading centres in a standardised manner. However, the extent of MVAs and their appearance in the retinal periphery are remaining unclear especially with SRT using microcollimated X-ray.

Therefore, the aim of this study was to provide a report about longer term efficacy and safety (including peripheral fluoresceine angiography and SD-OCT evaluation) of an SRT/TER combination therapy in $\mathrm{NAMD}$ in a clinical routine setting.

\section{MATERIALS AND METHODS}

This retrospective study followed 50 consecutive patients regarding visual acuity and effective treatment intervals up to 36 months and included an extensive imaging safety analysis after about 2 years. In all patients, SRT was performed by EyeRad Swiss Medical Center Binningen, Switzerland, within clinical routine between August 2013 and November 2014. The study and all its documents followed the tenets of the Declaration of Helsinki, International Council for Harmonisation - Good Clinical Practice guidelines and Swiss law; the protocol was reviewed and approved by the local ethics committee (Ethikkommission Nordwestschweiz; EKNZ No 2015-251). According to local requirements, general informed consent regarding retrospective analyses of data and use of imaging material was obtained from all patients.

Following the recommendations of INTREPID ${ }^{24}$ the initial patient selection criteria for SRT included: persistent nAMD activity despite 4-weekly anti-VEGF injections or necessity for frequent injections (4-weekly or 6-weekly); lesion within the central $4 \mathrm{~mm}$ circle; no advanced fibrosis or pigment epithelium atrophy zones as evaluated by OCT; $20 \mathrm{~mm}<$ axial length of bulb $<26 \mathrm{~mm}$; and ability to sit quietly with a slightly bowed head for approximately 30-60 min. ${ }^{17}$ Patients with diagnosis of any concurrent retinal vasculopathy were excluded. Patients were recruited from a clinical routine TER setting (see further). Single session SRT was performed using the IRay System (Carl Zeiss Meditec AG Germany) within the patient's specific anti-VEGF treatment interval, mostly within the last 2 weeks before next injection. The three sequential beams converge on the retina, delivering a $16 \mathrm{~Gy}$ dose to an area $4 \mathrm{~mm}$ in diameter, centred on the fovea. This non-invasive system comprises a precisioncontrolled X-ray tube, a patient interface, an eye stabilising device, an eye-tracking system, a graphical user interface and software for treatment planning. The system ${ }^{15}$ and the procedure in our setting ${ }^{17}$ have been described in full previously and were approved for clinical use in Europe.

The anti-VEGF pretreatment and follow-up at Vista Klinik Binningen, Switzerland, followed a strict TER. ${ }^{6}$ Within this protocol, anti-VEGF injections were performed due to a standard procedure ${ }^{25}$ at each visit. If no signs of intraretinal or subretinal fluid were observed on OCT or had remained stable for three consecutive visits and no new haemorrhage was visible, treatment intervals were sequentially lengthened by 2 weeks, from a starting interval of 4 weeks to a maximum of 12 weeks. In the case of OCT observed instability (new or increasing fluid) or new haemorrhage, treatment intervals were shortened by 4 weeks to a minimum of 4 weeks. If no recurrence or instability was shown with the maximum interval of 12 weeks, this was repeated two further times (in total $3 \times 12$ weeks) followed by 2 -monthly observations (if stable within all three 12 -week intervals).

Safety diagnostics was performed between April and September 2016 on both eyes including:

- SD-OCT (Spectralis, Heidelberg Eng, Germany) at Macular Volume Scan setting at High-Speed Mode, $20^{\circ} \times 20^{\circ}, 49$ sections, 15 frames.

- ETDRS seven-field colour stereoscopic pairs of photographs (FF450, Zeiss, Germany).

- Fluoresceine angiography (FA) using a standardised study protocol with $30^{\circ}$ settings in the seven ETDRS fields and additionally imaging of the periphery with a $55^{\circ}$ setting in eight directions of gaze (HRA2, Heidelberg Eng., Germany).

Masked evaluations of SD-OCT, colour fundus (CF) and FA images followed standard reading protocols at the Vienna Reading Center, an independent digital reading centre. Validated computer-assisted grading software was used, and the readers were trained according to the Vienna Reading Center protocol and received regular supervision by the grading leaders.

\section{Data analysis}

Data are presented as mean or percentage \pm SD. Differences between baseline and follow-up visits were tested for statistical significance using paired t-tests. $P$ values (two sided) were considered significant if $\mathrm{p}<0.05$. All statistical analyses were performed using SPSS V.27.0 for Windows.

\section{RESULTS}

We evaluated 50 eyes of 50 consecutive patients. Mean age was $78.2 \pm 6.7$ years, and $31(62 \%)$ patients were female. All patients had a history of anti-VEGF pretreatment prior SRT $(37.6 \pm 21.4$ months; 26.1 \pm 2.4 anti-VEGF pretreatments; 60\%/40\% receiving aflibercept/ranibizumab). For detailed baseline characteristics, see Hatz et al. ${ }^{17}$ The previous to SRT used anti-VEGF TER was continued during follow-up: for 48/50 eyes follow-up was available up to 36 months after SRT, and 2/50 eyes were lost to follow-up between 24 and 36 months. All patients attended a safety visit $24 \pm 4$ months (range 18-33 months) after SRT.

\section{Visual acuity and treatment intervals}

Best-corrected visual acuity (BCVA) remained stable during the

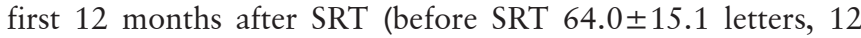
months $63.6 \pm 16.2$ letters, $\mathrm{p}=0.744)$ but decreased afterwards. The BCVA change was $-5.8 \pm 15.9$ and $-11.0 \pm 20.1$ letters at 24 and 36 months, respectively (figure 1). Eleven of $50(22 \%)$ and 16/48 (33\%) eyes lost $\geq 15$ letters (three lines) at 24 and 36 months, respectively. Among the $\geq 15$ letters losers the great majority were pseudophacic, while only in three and five eyes, respectively, the vision loss was partly attributable to cataract progression. Only two eyes gained $\geq 15$ letters at 24 months (both after cataract surgery during follow-up) and three eyes at 36 months (2/3 after cataract surgery).

Mean maximum recurrence-free treatment interval (RFTI) was significantly increased from $4.24 \pm 0.66$ weeks before SRT to $7.52 \pm 3.05$ weeks at 12 months $(\mathrm{p}<0.001)$ and afterwards remained stable at 24 and 36 months $(7.40 \pm 3.17 / 6.89 \pm 3.00$ weeks, $p=0.746 / 0.175)$. At 36 months, three patients had reached our TER exit criterion $(3 \times$ stable 12 weeks interval). However, at 36 months, $16 / 48$ eyes (33\%) had the same maximum RFTI like before SRT or shorter. Of these, six eyes never reached a longer RFTI during the follow-up, while the others had shown longer RFTIs at 12 or 24 months. In total, 25/50 eyes (50\%) did 
Mean Visual Acuity (ETDRS letters)

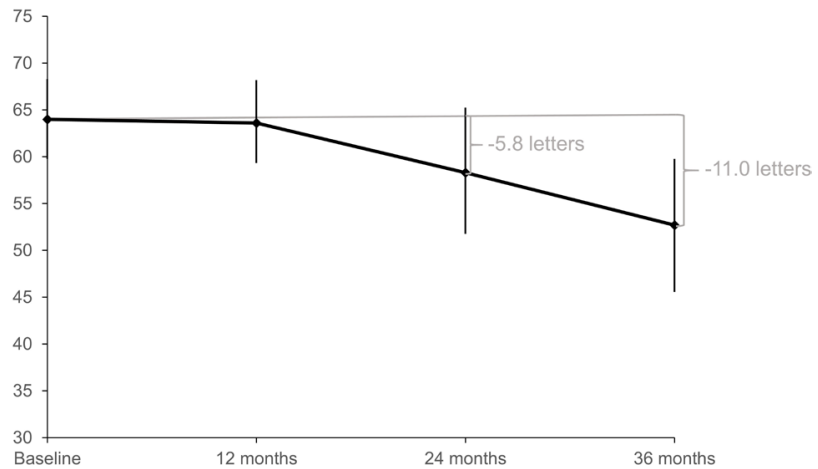

Figure 1 BCVA changes (with 95\% Cls) during follow-up: baseline refers to SRT; all patients were anti-VEGF pretreated. anti-VEGF, anti-vascular growth factor; BCVA, best-corrected visual acuity; SRT, stereotactic radiotherapy.

not reach anymore their individual maximum RFTI (reached at earlier follow-ups) at last evaluated visit.

\section{MVAs at colour fundus photography (CF) and FA}

Twelve months after SRT, there were no signs of MVAs due to $\mathrm{RR}$ at biomicroscopic examination. Reading centre evaluation of standardised CF of the $24 \pm 4$ month safety visit revealed cotton wool spots in $17 / 50$ (34\%) SRT eyes but in none of the fellow eyes. Twelve per cent SRT eyes showed dilated or tortuous vessels, $6 \%$ microaneurysms and $6 \%$ retinal vessels sheathing or narrowing (none of these changes in fellow eyes). None of the latter MVAs have been seen in eyes without cotton wool spots. For example, see figure 2. The majority of MVAs occurred in the nasal and/or inferior outer ETDRS grid subfield and/or the upper part of field 5 (figure 3). Thirteen of 50 (26\%) SRT eyes showed haemorrhages, four of these without other RR signs and therefore rather attributable to choroidal neovascularisation activity. For detailed findings/location, see table 1 .

Autofluorescence imaging revealed geographic atrophy (GA) in $24 / 50(48 \%)$ SRT eyes (mean area $3.901 \pm 7.005 \mathrm{~mm}^{2}$ ) and in $17 / 50(34 \%)$ fellow eyes $\left(9.746 \pm 16.822 \mathrm{~mm}^{2}\right)$. While 10 SRT eyes showed GA without having GA in the fellow eye, only three fellow eyes showed GA without GA in the SRT eye. In 48/50 patients, FA was performed, one patient did not agree and in one patient FA was not performed due to significantly reduced general condition and a history of multiple allergies. For detailed FA findings and their locations, see table 1. Nine of 48 (19\%) patients showed capillary non-perfusion areas (mean size within central ETDRS grid $2.911 \pm 2.118 \mathrm{~mm}^{2}$ ), of these four within the central $1 \mathrm{~mm}$ ETDRS subfield. For example, see figure 2. Only one eye with capillary non-perfusion at FA did not show changes at CF; the others had also MVAs at CF. Most changes were located in the inner and outer inferior and nasal ETDRS subfields. Peripheral FA did not reveal any MVAs outside the ETDRS fields 1-7. Taking together the results of CF and FA, 18/50 (36\%) SRT eyes showed MVAs (without haemorrhages). There was no significant difference in mean BCVA change between eyes without and with MVAs at 24 and 36 months $(-5.4 \pm 18.2$ vs $-6.4 \pm 11.4$ letters, $\mathrm{p}=0.831 ;-9.7 \pm 19.2$ vs $-13.4 \pm 21.9$ letters, $\mathrm{p}=0.545$ ).

\section{SD/OCT findings}

Mean central retinal thickness decreased from $407.3 \pm 153.2 \mu \mathrm{m}$ before SRT to $320.2 \pm 112.1 \mu \mathrm{m}$ at 12 months and afterwards
A1

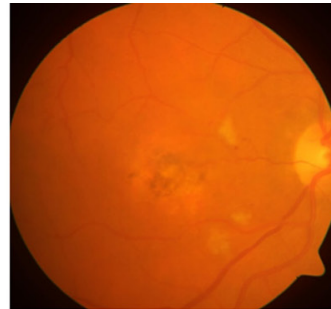

A2

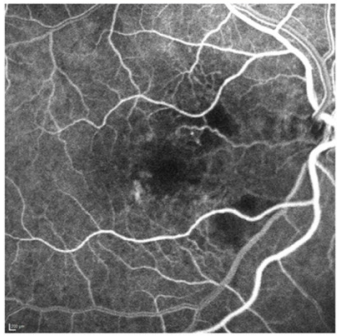

A3

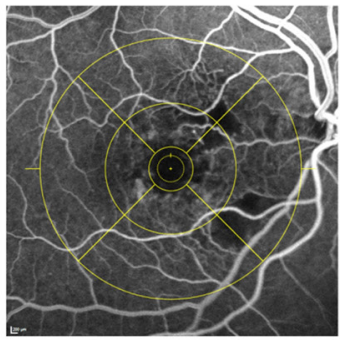

A4

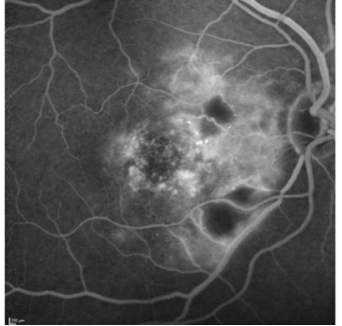

B1

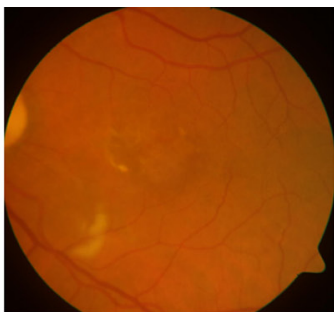

B2

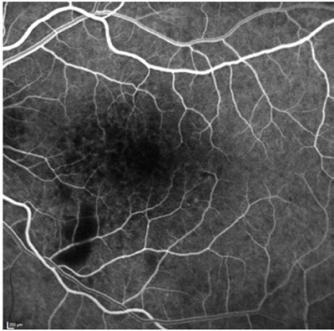

B3

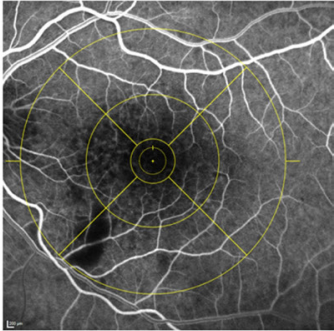

B4

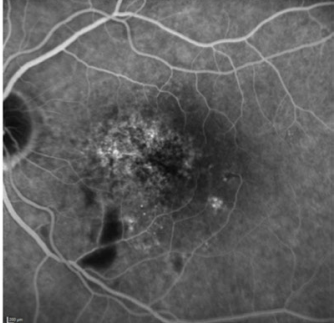

Figure 2 Example of microvascular abnormalities in two patients ( $A$ and $B$ ): colour fundus ETDRS F2 image (A1 and B1), FA ETDRS F2 early phase (A2 and B2), FA ETDRS F2 early phase with central ETDRS grid ( $A 3$ and $B 3$ ) and FA ETDRS F2 late phase (A4 and B4). Both patients with cotton wool spots (CF) and blockade due to cotton wool spots (FA). Patient A shows further microaneurysms, haemorrhages and vessel abnormalities (CF) as well as capillary non-perfusion, microaneurysms and retinal vessel leakage and calibre abnormalities (FA). Visual acuity was 55 (A) and 65 (B) letters at baseline, visual acuity loss was 20 letters (A) and 0 letters (B) at 36 months. CF, colour fundus photography; FA, fluoresceine angiography.

remained stable at $316.9 \pm 135.1 \mu \mathrm{m}$ at 24 and $307.3 \pm 124.3 \mu \mathrm{m}$ at 36 months $(p=0.8416, p=0.5826)$. The safety evaluation after $24 \pm 4$ months revealed a 'dry retina' (no intraretinal or subretinal fluid) in 36\%, intraretinal cysts in 44\% and subretinal fluid in 38\% of eyes; $18 \%$ showed both. For AMD specific OCT findings and their location, see online supplemental table 1.

Comparison of retinal layer thicknesses within the nine ETDRS grid subfields between baseline (before SRT) and safety visit showed a significant decrease in the central $1 \mathrm{~mm}$ subfield and the central point of the following layers: entire retina (inner limiting membrane (ILM) to retinal pigment epithelium (RPE)), outer retina (inner-outer segment junction (IS-OS) to RPE) and the nerve fibre layer (NFL) (ILM to outer border of NFL). For the entire retina significance for the decrease from baseline was further reached in the inner and outer inferior subfields as well as the inner nasal subfield. The NFL (ILM to outer border of 


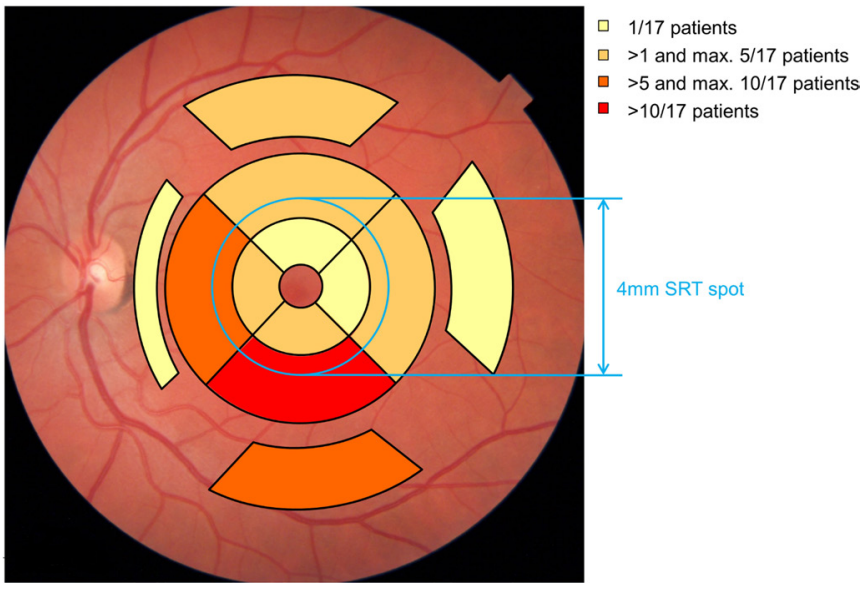

Figure 3 Distribution of microvascular abnormalities (CF) in relation to the SRT spot size. CF, colour fundus photography; SRT, stereotactic radiotherapy.

NFL) showed a significant decrease within its thickest area, the outer nasal subfield, representing parts of the papillomacular bundle, and a non-significant trend in the directly neighbouring inner nasal subfield. Among all ETDRS subfields the inner and outer basal subfields were most affected by a thickness decrease. For detailed results and $\mathrm{p}$ values, see table 2 .

\section{DISCUSSION}

This real-life but reading centre based safety analysis $24 \pm 4$ months after single session SRT in continuously anti-VEGF treated nAMD eyes revealed MVAs in 36\% of SRT eyes, most frequently located in the parafoveal inferior parts and accompanied by reduction of retinal layer thicknesses in the same areas and a possible loss in nerve fibre thickness in the papillomacular bundle. At 36 months, we found a mean reduction in visual acuity of about two lines and still prolonged treatment intervals in a TER compared with prior SRT.

Comparing our visual acuity results with INTREPID ${ }^{16} 26$ (for 16 Gy group -10.0 and -20 letters at 2 and 3 years, respectively), in our study, the loss from baseline was less severe $(-5.8 \pm 15.9$ and $-11.0 \pm 20.1$ letters). The continued anti-VEGF treatment using TER might have improved the visual acuity outcome in our population compared with the PRN treated INTREPID population. The metaanalysis by Kim et $a l^{5}$ favours TER in comparison with PRN regarding visual acuity outcome. The good treatment adherence with TER that combines each evaluation visit with an injection is also reflected by the stable mean treatment intervals through years 2 and 3 in our study. However, comparison of SRT and anti-VEGF combined treatment with long-term anti-VEGF monotherapy suggests a worse visual acuity outcome with the combined treatment. Recent long-term analyses of monotherapy revealed much more favourable visual acuity outcomes, ${ }^{27} 28$ which argues against the course of disease itself as only reason for this significant vision loss between years 1 and 3 in our population. However, comparison across studies is always problematic due to differences in case selection and so on and needs to be interpreted with considerable caution. Furthermore, our study showed no significant difference regarding visual acuity loss between eyes with MVAs and without MVAs, which might either be caused by our rather low patient number or it could be an argument against MVAs as reasons for visual acuity loss.

In our population, 12 months after SRT, there were no signs of RR at biomicroscopic fundus examination, ${ }^{17}$ while after $24 \pm 4$ months (range 18-33 months), 36\% of SRT eyes showed MVAs in CF and/or FA. It remains unclear if FA would have revealed any early MVA changes if it was performed after 12 months. The interval until diagnosis of MVAs after radiotherapy for nAMD varies quite much but has been shown to be less than 12 months only rarely. ${ }^{2326}$ The frequency of MVAs in our real-life setting was comparable with this in INTREPID year $3(30.3 \%)$

Table 1 Microvascular abnormalities within the central ETDRS grid (total, central $1 \mathrm{~mm}$, inner ring, outer ring) and outside grid at CF and FA

\begin{tabular}{|c|c|c|c|}
\hline Finding & Percentage of patients & Major locations & Further locations \\
\hline \multicolumn{4}{|l|}{$\begin{array}{l}\text { Colour fundus photography (CF) } \\
(n=50)\end{array}$} \\
\hline Cotton wool spots & 34 & Outer inferior and nasal ETDRS grid subfields. & Inner inferior ETDRS grid subfield: F5, F3, F4 and F1. \\
\hline Microaneurysms & 6 & Outer inferior ETDRS grid subfield. & Outer nasal and inferior ETDRS grid subfields: F5. \\
\hline Dilated or tortuous vessels & 12 & Outer inferior and nasal ETDRS grid subfields. & $\begin{array}{l}\text { All remaining ETDRS grid subfields (except } \mathrm{cmm} \text { ): F5, } \\
\text { F4 and F1. }\end{array}$ \\
\hline Retinal vessel sheating or narrowing & 6 & Outer inferior ETDRS grid subfield. & $\begin{array}{l}\text { All remaining ETDRS grid subfields (except } \mathrm{cmm} \text { ): } \\
\text { F5 and F4. }\end{array}$ \\
\hline Haemorrhages & 26 & Outer inferior and nasal ETDRS grid subfields. & $\begin{array}{l}\text { All remaining ETDRS grid subfields (except cmm): F5, } \\
\text { F3, F4 and F1. }\end{array}$ \\
\hline \multicolumn{4}{|l|}{ Fluoresceine angiography (FA) ( $n=48)$} \\
\hline Capillary non-perfusion & 19 & $\begin{array}{l}\text { Central and inner and outer inferior and inner and } \\
\text { outer nasal ETDRS grid subfields. }\end{array}$ & All remaining ETDRS grid subfields: F5, F4 and F1. \\
\hline Retinal vessel staining or leakage & 25 & $\begin{array}{l}\text { All inner and outer inferior and nasal ETDRS grid } \\
\text { subfields. }\end{array}$ & $\begin{array}{l}\text { All remaining ETDRS grid subfields (except } \mathrm{cmm} \text { ); } \mathrm{F5} \text {, } \\
\text { F4 and F1. }\end{array}$ \\
\hline Microaneurysms & 15 & Outer inferior and nasal ETDRS grid subfields & $\begin{array}{l}\text { Inner inferior and nasal and remaining outer ETDRS } \\
\text { grid subfields: F5, F3, F4 and F1. }\end{array}$ \\
\hline Dilated or tortuous vessels & 27 & $\begin{array}{l}\text { Inner and outer inferior and inner and outer nasal } \\
\text { ETDRS grid subfields. }\end{array}$ & $\begin{array}{l}\text { All remaining ETDRS grid subfields (except } \mathrm{cmm} \text { ): } \\
\text { F5 and F1. }\end{array}$ \\
\hline Retinal vessel narrowing & 27 & $\begin{array}{l}\text { Inner and outer inferior and inner nasal ETDRS grid } \\
\text { subfields. }\end{array}$ & $\begin{array}{l}\text { All remaining ETDRS grid subfields (except } \mathrm{cmm} \text { ): } \mathrm{F5} \text {, } \\
\mathrm{F} 3, \mathrm{~F} 4 \text { and } \mathrm{F} 1 \text {. }\end{array}$ \\
\hline Blockade due to haemorrhages & 15 & Outer nasal ETDRS grid subfield. & $\begin{array}{l}\text { All remaining ETDRS grid subfields (except } \mathrm{cmm} \text { ): F5, } \\
\text { F3 and F4. }\end{array}$ \\
\hline
\end{tabular}


but higher than the INTREPID 2-year data (13.1\%). ${ }^{26}$ Unfortunately, there is no other 2-year and 3-year data regarding MVAs after SRT available. Differences might be caused by our later FA/ CF evaluation at least in a few cases (range 18-33 months) than in INTREPID 2 years, the higher percentage of patients lost to follow-up in INTREPID and differences in imaging modalities and evaluation of RR-related changes.

Interestingly, the most frequent locations of MVA in the parafoveal inferior and nasal regions were exactly the same in this real-life analysis and in INTREPID. ${ }^{26}$ As these are the only both angiographic evaluations beyond the first year after SRT, this consistency might attract our attention. Furthermore, these findings were supported by significant decreases of layer thicknesses of the entire retina and the ganglion cell layer-inner plexiform layer-inner nuclear layer in the inner and outer inferior subfields of central ETDRS grid. One may speculate of either delivery of a higher dose to these areas compared with others or an increased sensitivity of these areas. For the used IRay System, 90th isodose curves have been described and correspond to a $4 \mathrm{~mm}$ spot size centred in the macula. ${ }^{29}$ Hanlon et $a l^{30}$ demonstrated for 32 eye models that the therapeutic dose, which is delivered by three divergent photon beams entering through the sclera from inferior and overlapping on the macula, only fluctuated $<6 \%$ in the clinical target volume. As the targeting was assisted by immobilising the eye and by use of a tracking system gaze deviations were minimised..$^{29}$ Anyway, the latter are unlikely to cause such regularity in questionable higher delivery to certain areas. But the entrance of the three beams from inferior might be an aspect to evaluate. Hanlon et $a l^{30}$ showed the asymmetric dose distribution produced by the IRay system in three-dimensional CT reconstructions of eye models. A larger volume of the inferior part of the retina is exposed to higher doses compared with the upper part. This is explained by the entrance of all three beams from the inferior sclera to avoid a relevant dose to the radiosensitive lens. Compared with the steep dose decrease achievable by beta-sources, this may in part explain the more frequent MVAs. This larger treatment volume has been the handicap in all external beam techniques used so far, either photon or proton beams.

Other than the upper and lower regions of the macula without any known anatomical difference, the parafoveal nasal region is this one with the thickest NFL. As the most MVAs we detected were cotton wool spots in CF representing localised swelling in the NFL, it could be hypothesised that many of these are found in the region with the most nerve fibres.

Like for MVAs at CF and FA, it can be assumed that radiationspecific OCT changes might occur during later follow-up. Therefore, it seems not surprising that the peripapillary NFL thickness did not show a significant decrease during the first 12 months after SRT in an nAMD case series. ${ }^{31}$ In our reading centre based study, a significant decrease in nerve fibre thickness within the outer nasal subfield of central ETDRS grid, representing parts of the papillomacular bundle, and a non-significant trend in the directly neighbouring inner nasal subfield were found after $24 \pm 4$ months. Despite this cannot directly be compared with peripapillary thickness measurements using an automated peripapillary ring scan modus, it might indicate a nerve fibre loss in the papillomacular bundle in combined treated eyes over the long term. It has recently been shown that intravitreal injection monotherapy did not affect peripapillary retinal NFL at least during the first year of treatment, ${ }^{32}$ while others did not exclude an effect over 2.5 years. ${ }^{33}$ However, together with the finding of parafoveal nasal MVAs, the issue of possible nerve fibre loss in the papillomacular bundle should be followed further. 
SRT was introduced with the aim to reduce the anti-VEGF treatment frequency and therefore the burden to the patient and to the healthcare system. ${ }^{34}$ Patients expected fewer intravitreal injections and reported positive experiences of receiving SRT treatment. ${ }^{35}$ Results of 1-year or 2-year data consistently supported that a reduction in injection frequency could significantly be reached in pretreated ${ }^{161736}$ and treatment-naïve $\mathrm{nAMD}^{37}$ patients by adding a single session SRT to anti-VEGF standard therapy. However, as INTREPID was designed as a 2-year trial and already published real-life data only cover the first year, our analysis is the first evaluating treatment frequency in the third year. The mean maximum RFTI in our population remained stable at 24 and 36 months compared with the 12 months' value, which had almost doubled from baseline. Further evaluation revealed that $50 \%$ of eyes did not reach anymore their individual maximum RFTI (reached at earlier follow-ups) at the 36-month follow-up. Therefore, a weakening of the SRT effect on nAMD activity in a few patients within the third year cannot be excluded.

Finally, the optimal treatment schedule and dose should be discussed. Reflecting the long-term experiences from singlefraction stereotactic radiation therapy (radiosurgery) in other benign conditions (arteriovenous malformation; Schwanoma), an increase of the stereotactic dose from 12 to more than $18 \mathrm{~Gy}$ has increased the risk of late side effects. ${ }^{38}$ However, doses of $12 \mathrm{~Gy}$ in single fractions have been proven sufficient in different types of benign vascular diseases. ${ }^{38} 39$ So, using biological models to estimate the optimal dose as a compromise of long-term efficiency and avoidance of late effects, a fraction size of about 12 Gy may be optimal and should be tested in nAMD. This dose is more efficient than the initially used fractionated schedules for $\mathrm{nAMD}^{18}$ (ie, 10 times $2 \mathrm{~Gy}$ ) but is lower than the $16 \mathrm{~Gy}$ being set as standard, the latter causing some late effects to the retina.

Strengths of our study are the reading centre based standardised image analysis for CF, FA and SD-OCT including SD-OCT layer analyses, standardised study protocol imaging plus angiography of the retinal periphery, the safety assessment in all included patients, the high follow-up rate for efficacy analysis at year 3 and the continuous anti-VEGF TER treatment. Limitations are its retrospective nature, the range regarding the moment of safety analysis and the lack of a control group. Due to the retrospective setting, the dedicated population (see Methods) and the variety of $\mathrm{AMD}$, an adequate matching control group was not possible, and therefore, the outcome parameters were compared with the pre-SRT period. Nowadays, OCT angiography, which was not yet available at our site at the time point of safety analyses, might have added further information and would have been the ideal non-invasive follow-up method.

In summary, SRT as adjunct therapy to anti-VEGF intravitreal injections for nAMD in a real-life setting provided an extension of the anti-VEGF treatment interval through year 3 despite a significant loss in visual acuity compared with prior SRT. After about 2 years, a remarkable part of patients show MVAs that might be attributable to SRT and are most frequently located in the parafoveal inferior and nasal region. Furthermore, the NFL thickness might decrease in the papillomacular bundle. Due to these findings, a further close follow-up of all SRT patients is recommended, and the use of SRT with the described settings must be seen critically.

Funding This study has been supported in part by Carl Zeiss Meditec AG Germany. The authors would like to thank the study coordinator Mrs Susanne Mueller and the chief photographer Mrs Christine Knodel for their support.
Disclaimer The industry contributor has had no role in the study and the manuscript.

Competing interests This study was supported in part by Carl-Zeiss Meditec AG, Germany (grant to KH, Vista Klinik). Carl-Zeiss Meditec AG, Germany, did not have any influence on the study protocol, study realisation, data analysis and interpretation.

\section{Patient consent for publication Not required.}

Ethics approval The protocol was reviewed and approved by the local ethics committee (Ethikkommission Nordwestschweiz; EKNZ No 2015-251).

Provenance and peer review Not commissioned; externally peer reviewed.

Data availability statement Data are available on reasonable request. Patient data have been obtained in routine treatment and have been blinded. The patients have signed a general consent form.

Supplemental material This content has been supplied by the author(s). It has not been vetted by BMJ Publishing Group Limited (BMJ) and may not have been peer-reviewed. Any opinions or recommendations discussed are solely those of the author(s) and are not endorsed by BMJ. BMJ disclaims all liability and responsibility arising from any reliance placed on the content. Where the content includes any translated material, BMJ does not warrant the accuracy and reliability of the translations (including but not limited to local regulations, clinical guidelines, terminology, drug names and drug dosages), and is not responsible for any error and/or omissions arising from translation and adaptation or otherwise.

Open access This is an open access article distributed in accordance with the Creative Commons Attribution Non Commercial (CC BY-NC 4.0) license, which permits others to distribute, remix, adapt, build upon this work non-commercially, and license their derivative works on different terms, provided the original work is properly cited, appropriate credit is given, any changes made indicated, and the use is non-commercial. See: http://creativecommons.org/licenses/by-nc/4.0/.

\section{ORCID iDs}

Katja Hatz http://orcid.org/0000-0003-2807-0310

Ursula Margarethe Schmidt-Erfurth http://orcid.org/0000-0002-7788-7311

Bianca S Gerendas http://orcid.org/0000-0001-8940-8130

\section{REFERENCES}

1 Pascolini D, Mariotti SP, Pokharel GP, et al. 2002 global update of available data on visual impairment: a compilation of population-based prevalence studies. Ophthalmic Epidemiol 2004;11:67-115.

2 Campbell JP, Bressler SB, Bressler NM. Impact of availability of anti-vascular endothelial growth factor therapy on visual impairment and blindness due to neovascular age-related macular degeneration. Arch Ophthalmol 2012;130:794-5.

3 Holz FG, Tadayoni R, Beatty S, et al. Multi-country real-life experience of anti-vascular endothelial growth factor therapy for wet age-related macular degeneration. $\mathrm{Br} \mathrm{J}$ Ophthalmol 2015;99:220-6

4 Comparison of Age-related Macular Degeneration Treatments Trials (CATT) Research Group, Martin DF, Maguire MG, et al. Ranibizumab and bevacizumab for treatment of neovascular age-related macular degeneration: two-year results. Ophthalmology 2012;119:1388-98.

5 Kim LN, Mehta H, Barthelmes D, et al. METAANALYSIS of real-world outcomes of intravitreal ranibizumab for the treatment of neovascular age-related macular degeneration. Retina 2016;36:1418-31.

6 Hatz K, Prünte C. Treat and extend versus pro re NatA regimens of ranibizumab in neovascular age-related macular degeneration: a comparative 12 month study. Acta Ophthalmol 2017;95:e67-72.

7 Brown MM, Brown GC, Lieske HB, et al. Societal costs associated with neovascular age-related macular degeneration in the United States. Retina 2016:36:285-98.

8 van Asten F, Michels CTJ, Hoyng CB, et al. The cost-effectiveness of bevacizumab, ranibizumab and aflibercept for the treatment of age-related macular degeneration-A cost-effectiveness analysis from a societal perspective. PLoS One 2018;13:e0197670.

9 Hollingworth W, Jones T, Reeves BC, et al. A longitudinal study to assess the frequency and cost of antivascular endothelial therapy, and inequalities in access, in England between 2005 and 2015. BMJ Open 2017:7:e018289.

10 Spielberg L, Leys $A$. Treatment of neovascular age-related macular degeneration with a variable ranibizumab dosing regimen and one-time reduced-fluence photodynamic therapy: the Torpedo trial at 2 years. Graefes Arch Clin Exp Ophthalmol 2010:248:943-56

11 Hatz K, Schneider U, Henrich B, et al. Comparing ranibizumab monotherapy and combination with single photodynamic therapy in wet AMD: retreatment and morphologic results. Eur J Ophthalmol 2017:27:470-5.

12 Dugel PU, Petrarca R, Bennett M, et al. Macular epiretinal brachytherapy in treated age-related macular degeneration: MERITAGE study: twelve-month safety and efficacy results. Ophthalmology 2012;119:1425-31. 
13 Petrarca R, Dugel PU, Bennett M, et al. Macular epiretinal brachytherapy in treated age-related macular degeneration (MERITAGE): month 24 safety and efficacy results. Retina 2014;34:874-9.

14 Dugel PU, Bebchuk JD, Nau J, et al. Epimacular brachytherapy for neovascular age-related macular degeneration: a randomized, controlled trial (Cabernet). Ophthalmology 2013;120:317-27.

15 Jackson TL, Chakravarthy U, Kaiser PK, et al. Stereotactic radiotherapy for neovascular age-related macular degeneration: 52-week safety and efficacy results of the INTREPID study. Ophthalmology 2013;120:1893-900.

16 Jackson TL, Chakravarthy U, Slakter JS, et al. Stereotactic radiotherapy for neovascular age-related macular degeneration: year 2 results of the INTREPID study. Ophthalmology 2015;122:138-45.

17 Hatz K, Zimmermann F, Kardamakis D, et al. Low-Energy stereotactic radiotherapy for treatment of exudative age-related macular degeneration in a Treat-and-Extend regimen. Ophthalmic Surg Lasers Imaging Retina 2018;49:86-93.

18 Evans JR, Sivagnanavel V, Chong V. Radiotherapy for neovascular age-related macular degeneration. Cochrane Database Syst Rev 2010;12:CD004004.

19 Hart PM, Chakravarthy U, Mackenzie G, et al. Visual outcomes in the subfoveal radiotherapy study: a randomized controlled trial of teletherapy for age-related macular degeneration. Arch Ophthalmol 2002;120:1029-38.

20 Jackson TL, Desai R, Simpson A, et al. Epimacular brachytherapy for previously treated neovascular age-related macular degeneration (MERLOT): a phase 3 randomized controlled trial. Ophthalmology 2016;123:1287-96.

21 Giuliari GP, Sadaka A, Hinkle DM, et al. Current treatments for radiation retinopathy. Acta Oncol 2011;50:6-13.

22 Petrarca R, Dugel PU, Nau J, et al. Macular epiretinal brachytherapy in treated agerelated macular degeneration (MERITAGE): month 12 optical coherence tomography and fluorescein angiography. Ophthalmology 2013;120:328-33.

23 Jackson TL, Dugel PU, Bebchuk JD, et al. Epimacular brachytherapy for neovascular age-related macular degeneration (Cabernet): fluorescein angiography and optical coherence tomography. Ophthalmology 2013;120:1597-603.

24 Jackson TL, Shusterman EM, Arnoldussen M, et al. Stereotactic radiotherapy for wet age-related macular degeneration (INTREPID): influence of baseline characteristics on clinical response. Retina 2015;35:194-204.

25 Grzybowski A, Told R, Sacu S, et al. 2018 update on intravitreal injections: Euretina expert consensus recommendations. Ophthalmologica 2018;239:181-93.

26 Freiberg FJ, Michels S, Muldrew A, et al. Microvascular abnormalities secondary to radiation therapy in neovascular age-related macular degeneration: findings from the INTREPID clinical trial. Br J Ophthalmol 2019;103:469-74.
27 Haddad W-M, Minous FLE, Legeai J, et al. Long-Term outcomes and incidence of recurrence of neovascularization in treated exudative age-related macular degeneration. Retina 2017;37:951-61.

28 Gillies MC, Campain A, Barthelmes D, et al. Long-Term outcomes of treatment of neovascular age-related macular degeneration: data from an observational study. Ophthalmology 2015;122:1837-45.

29 Taddei PJ, Chell E, Hansen S, et al. Assessment of targeting accuracy of a low-energy stereotactic radiosurgery treatment for age-related macular degeneration. Phys Med Biol 2010;55:7037-54.

30 Hanlon J, Firpo M, Chell E, et al. Stereotactic radiosurgery for AMD: a Monte Carlo-based assessment of patient-specific tissue doses. Invest Ophthalmol Vis Sci 2011;52:2334-42.

31 Ranjbar M, Kurz M, Holzhey A, et al. Changes in peripapillary nerve fiber layer thickness after adjuvant stereotactic radiotherapy in patients with neovascular agerelated macular degeneration. Curr Eye Res 2017;42:1698-706.

32 Zucchiatti I, Cicinelli MV, Parodi MB, et al. Effect of intravitreal ranibizumab on ganglion cell complex and peripapillary retinal nerve fiber layer in neovascular agerelated macular degeneration using spectral domain optical coherence tomography Retina 2017:37:1314-9.

33 Enders P, Sitnilska V, Altay L, et al. Retinal nerve fiber loss in anti-VEGF therapy for age-related macular degeneration can be decreased by anterior chamber paracentesis Ophthalmologica 2017;237:111-8.

34 Neubauer AS, Reznicek L, Minartz C, et al. [Economic Short-Term Cost Model for Stereotactic Radiotherapy of Neovascular AMD]. Klin Monbl Augenheilkd 2016;233:951-7.

35 Senra H, Joseph S, Balaskas K, et al. Patient perceptions and experiences of stereotactic radiotherapy for wet age-related macular degeneration. Eur J Ophthalmol 2016;26:e80-2.

36 Ranjbar M, Kurz M, Holzhey A, et al. Stereotactic radiotherapy in neovascular agerelated macular degeneration: real-life efficacy and morphological evaluation of the outer retina-choroid complex. Medicine 2016;95:e5729.

37 Brand C, Arnoldussen M. IRay therapy as an adjuvant therapy in newly diagnosed patients with neovascular age-related macular degeneration. Eye 2018;32:1345-52.

38 Gilbo P, Zhang I, Knisely J. Stereotactic radiosurgery of the brain: a review of common indications. Chin Clin Oncol 2017;6:S14.

39 Lee CC, Trifiletti DM, Sheehan JP. Radiosurgery for glomus tumors. In: Niranjan A, Lunsford LD, Kano H, eds. Leksell radiosurgery. Basel, Karger: Prog Neurol Surg, 2019: 34. 215-22. 